\title{
EDITORIAL
}

\section{Regionale StadtLandschaften}

\author{
Sabine Hofmeister • Andreas Klee
}

Online publiziert: 16. März 2015

(C) Springer-Verlag Berlin Heidelberg 2015

Hier die pulsierende Stadt - dort das bäuerliche Land? Hier heruntergekommene Altbauquartiere - dort idyllische Dorfszenerien? Dichotomisierungen dieser Art, die in den meisten Fällen ohnehin konstruierte sind, gehören der Vergangenheit an. Sie haben sich zusehends in eine Vermischung verwandelt. Die früher noch wahrnehmbaren Grenzen zwischen Stadt und Land lösen sich immer mehr auf. Merkte man bis vor einem halben Jahrhundert noch deutlich, ob man in einer Stadt oder einem Dorf unterwegs war, ob man in einem ,gut bürgerlichen“ Restaurant aß oder in einem Dorfwirtshaus, ob man mit einer Städterin sprach oder mit einem „Dörfler“, so ist das heute wesentlich komplexer geworden. Landschaften scheinen zu verstädtern, Städte sind durchzogen von „Landschaften“ - von Freiräumen und naturnahen Flächen. Auch die Lebensweisen der Menschen gleichen sich zusehends mehr an.

Kurzum: Städte und Landschaften verändern sich hinsichtlich Physiognomie und Wahrnehmung, hinsichtlich Wirtschaften und Kultur, und vor allem hinsichtlich ihres vermeintlichen Gegensatzes. Die Raumkategorien „Stadt“ und „Land“ können nicht länger unabhängig voneinander gesehen werden. Sie überlagern und vermischen sich und bilden schließlich ein Konglomerat heraus, das man mit der

Dr. A. Klee $(\square)$

Akademie für Raumforschung und Landesplanung (ARL) -

Leibniz-Forum für Raumwissenschaften,

Hohenzollernstraße 11,

30161 Hannover, Deutschland

E-Mail: klee@arl-net.de

Prof. Dr.-Ing. S. Hofmeister

Leuphana Universität Lüneburg,

Scharnhorststraße 1,

21335 Lüneburg, Deutschland

E-Mail: hofmeister@uni.leuphana.de
Vokabel der „Regionalen StadtLandschaften“ gut umschreiben kann.

In den „Regionalen Stadtlandschaften“ zeigen sich alle der gegenwärtigen gesellschaftlichen und auch politischplanerischen Herausforderungen. Megatrends wie Globalisierung, demographischer Wandel, Pluralisierung der Lebensstile, Klimawandel und Energiewende prägen diese Regionen in differenzierter Weise. Die unterschiedlichen Ausprägungen sind nicht an politisch-administrativen Grenzen orientiert, sondern gleichen eher einem Mosaik. Diese Konstellationen haben auch Auswirkungen auf die Forschung. Eine disziplinübergreifende und raumspezifische Betrachtungsweise ist erforderlich, um den gegenwärtigen Wandel dieser „Regionalen StadtLandschaften“ angemessen zu analysieren, zu verstehen und zu gestalten.

Mit dem Wandel der Funktionsprinzipien und regionalen Besonderheiten von „Regionalen StadtLandschaften“, mit den Möglichkeiten, die Veränderungen zu gestalten und zu steuern, hat sich die Akademie für Raumforschung und Landesplanung (ARL) im Rahmen ihres Jahreskongresses 2013 intensiv beschäftigt. Im Juni 2013 kamen in Hamburg rund 200 Expertinnen und Experten zusammen, um über urbane und suburbane Räume einem integrierten Verständnis folgend zu diskutieren und dabei wirtschaftliche, soziale und ökologische Aspekte gemeinsam mit den landschaftlichen Qualitäten und Potenzialen zu denken, die es planerisch in Wert zu setzen gilt.

Drei Beiträge dieses Heftes basieren auf Vorträgen, die beim ARL-Kongress 2013 gehalten wurden. Cordula Kropp fragt in ihrem Beitrag ,Regionale StadtLandschaften Muster der lebensweltlichen Erfahrung postindustrieller Raumproduktion zwischen Homogenisierung und Fragmentierung", was es für die lebensweltliche Raumerfahrung bedeutet, wenn im Stadt-Land-Kontinuum die Attribute „ländlich“ und „städtisch“ immer weniger Aussagekraft 
haben. Sie greift dabei auf die Raumkonzepte von Henri Lefebvre zurück, der die postindustrielle Raumproduktion zwischen Homogenisierungstendenzen und Fragmentierungsprozessen skizziert. Cordula Kropp leitet daraus Muster der Raumerfahrungen ab, die „Zwischen unendlichen Möglichkeiten und wachsender Überforderung" charakterisiert werden können und sich dabei auf das gesamte Spektrum der urbane und ländliche Räume zusammenfassenden Raumdynamiken beziehen.

Den Fokus auf soziale und ökonomische Probleme in der „Regionalen StadtLandschaft" legen Henning Nuissl, Janko Vollmer, David Westenberg und Jan-Niklas Willing in ihrem Beitrag „Die Konzentration von Altersarmut in der StadtLandschaft - Probleme und Handlungsbedarfe". Sie nehmen die zunehmende demographische Alterung sowie das anhaltende Auseinanderdriften der sozialen Lagen in den Stadtregionen zum Anlass, über die Konsequenzen einer räumlichen Überlagerung dieser Entwicklungen nachzudenken. Lassen sich Anzeichen einer sozialräumlichen Polarisierung in Stadtregionen erkennen, die mit dem Ausdruck Altersarmut begrifflich gefasst werden kann? Welche Konsequenzen ergeben sich daraus für die gesellschaftliche Entwicklung und für die Stadt(planungs) politik? Die Autoren plädieren unter anderem für eine sozialraumorientierte Stadt- und Quartiersentwicklung, die soziale Unterstützungsnetzwerke und informelle Strukturen zusammenbindet.

Stefanie Rößler widmet sich in ihrem Beitrag der „klimawandelgerechten Stadtentwicklung durch grüne Infrastruktur" und greift damit einen zentralen Bestandteil kommunaler und regionaler Klimaanpassungsstrategien heraus. Sie diskutiert verschiedene planerische Ansätze freiraumplanerischer Maßnahmen, die die grüne Infrastruktur in Stadtregionen in Wert setzen können, um die Folgen des Klimawandels besser bewältigen zu können. Ihre Ergebnisse fußen auf dem „Integrierten Regionalen Klimaanpassungsprogramm für die Region Dresden“" und liefern insbeson- dere auch der planenden Verwaltung wichtige Anregungen im Umgang mit dem Klimawandel.

Abgerundet wird diese Ausgabe zum einen durch den Beitrag von Jens Hoffmann, Peter Dehne, Thomas Weith, Christian Strauß und Nadin Gaasch, der das Heft eröffnet. Sie fragen, ob der demographische Wandel in Deutschland in direkter Weise zu dem seit Längerem zu beobachtenden Landnutzungswandel beiträgt. Hierzu haben die Autoren eine Reihe von Veröffentlichungen und Studien ausgewertet und diskutierten die hierbei gewonnenen Erkenntnisse mit Expertinnen und Experten. Es wurde deutlich, dass die gestellte Frage aufgrund der Komplexität und Schwierigkeit der Analyse, Bewertung und Abbildung von Zusammenhängen zwischen demographischem Wandel und Landnutzungswandel nur schwer zu beantworten ist. Aber es eröffnen sich vielfältige Möglichkeiten zur Qualifizierung von Steuerungsansätzen. Die Autoren kommen zum Schluss, dass der demographische Wandel ein „kommunikative(s) Einstiegsthema" darstellt, mit dem Landnutzungsänderungen und Landnutzungskonflikte diskutiert und auf die politische Agenda gesetzt werden können.

Zum anderen stellt Thomas Uhlendahl in seinem Bericht „Bürgerbeteiligung zur Gemeindeentwicklung“ Ansatz, Methoden und Ergebnisse einer neuen Form der kommunalen Entwicklungsplanung unter besonders intensiver Beteiligung von Bürgerinnen und Bürgern vor. Am Beispiel der Gemeinde Steinach im Kinzigtal (Baden-Württemberg) zeigt der Autor, wie es gelingen kann, auch Bevölkerungsgruppen für Planungsprozesse zu interessieren, die üblicherweise kaum Zugang zu Planungsfragen haben. So geschah es in Steinach, wo ein vom Land geförderter „BürgerInnenRat" in einem breit angelegten Dialogprozess Ergebnisse erarbeitet hat, die in das Gemeindeentwicklungskonzept direkt eingeflossen sind. Wie im Beitrag ausgeführt wird, ist das Verfahren zur Nachahmung empfohlen.

Wir wünschen allen Leserinnen und Lesern eine anregende Lektüre. 